\title{
Digital Modulation and Demodulation Technique of FSK
}

\section{Wei Yang ${ }^{a^{*}}$, Yuwen Wang ${ }^{b}$, Yongwei Lic and Yingzhu Chen ${ }^{d}$} School of Aeronautics and Astronautics, University of Electronic Science and Technology of China,
Chengdu 611731, China;

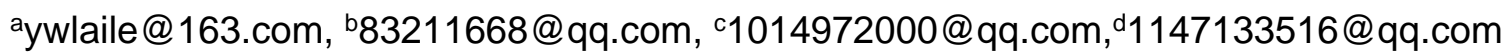

Keywords: 2FSK, MFSK, Modulation, Demodulation.

\begin{abstract}
Digital modulation technology is widely applied. The principle of Binary Frequency-Shift Keying (2FSK) modulation is introduced and it is extended to Multi-Frequency-Shift Keying (MFSK). In this paper, a simple and easy modulation and demodulation method is presented. Frequency modulation uses baseband digital signal to control the number of sinusoidal signal per cycle data. Frequency demodulation uses the peak value of each group of signals to determine the corresponding baseband signals. The effectiveness of the proposed method is verified by simulation experiments.
\end{abstract}

\section{Introduction}

Most channels are not directly used for baseband signal transmission. The baseband signal is used to control some parameters of the carrier wave, which makes the parameters change with the change of the baseband signal, this is the so-called signal modulation. In principle, the modulated carrier wave can be arbitrary waveform, as long as it is suitable for medium transmission, and can distinguish between different signals [1]. In fact, in most digital communication systems, the sine signal is chosen as the carrier, which is simple and easy to generate and receive. Compared with analog modulation and digital modulation, the principle is basically the same, the difference is: in the process of analog modulation, the parameters of the carrier signal are continuously modulated, at the receiving end, the parameters of the carrier signal are continuously evaluated.

\section{FSK Model}

Carrier frequency modulation with digital signal is called frequency shift keying (FSK) [2]. It is widely used because of its strong anti-fading ability. FSK is used to transmit digital signals with different frequencies, and the frequency of the carrier signal is controlled by the digital baseband signal. The carrier signal received at the receiving end is converted into digital signal to complete the process of information transmission.

2FSK is the simplest form of frequency shift keying, which can be expressed as:

$S(t)=m_{1}(t) A \cos \left(2 \pi f_{1} t+\varphi_{1}\right)+m_{2}(t) A \cos \left(2 \pi f_{2} t+\varphi_{2}\right)$

In the formula, $m_{1}(t)=\sum_{n=-\infty}^{\infty} \bar{b}_{n} g\left(t-n T_{b}\right), m_{2}(t)=\sum_{n=-\infty}^{\infty} b_{n} g\left(t-n T_{b}\right), A$ is the amplitude of the carrier, $T_{b}$ is the period of digital symbol, $b_{n}$ is a sequence of numbers, $\bar{b}_{n}$ is the complement of $b_{n}$.

Multi-frequency modulation model can be formed by adding sine wave with different numbers, different frequency and amplitude. For example,

$$
\begin{array}{r}
S(t)=m_{1}(t) A \cos \left(2 \pi f_{1} t+\varphi_{1}\right)+ \\
m_{2}(t) A \cos \left(2 \pi f_{2} t+\varphi_{2}\right)+ \\
m_{3}(t) A \cos \left(2 \pi f_{3} t+\varphi_{3}\right)+ \\
\vdots \\
m_{m}(t) A \cos \left(2 \pi f_{m} t+\varphi_{m}\right)+
\end{array}
$$




\section{Carrier Signal}

Sine wave can be used as carrier signal. First create a complete cycle of sine wave. Move the sine wave to 0 to 2, and then extend it to 0 to 255 . Finally, round the sine wave. C language program is as follows:

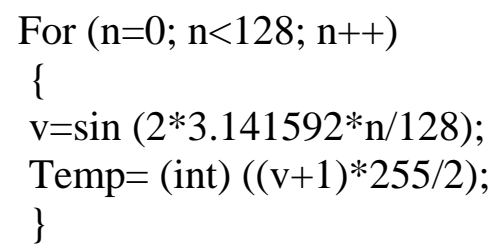

The above program generates a 128*8 bit sine wave. Import data of the sine wave into MIF file. Use Quartus II to develop a 128*8 bit ROM, Then add the MIF file to ROM. There are two ways to change carrier frequency:

a. Improve the clock frequency. The speed of reading data from ROM will speed up, and the time interval between data points will be shortened. The time used to read data from a cycle is shortened, so the period of output sine signal is small and the frequency is increased.

b. The clock frequency remains the same, but reduce the number of reading data. For example, the interval of a point to read the data, the original cycle data has 128 , now only 64 , the output signal frequency doubled.

In this paper, we use the method of reducing the number of data to realize the change of carrier frequency. Each frequency corresponds to a baseband digital signal. Baseband signal to control the carrier frequency, so that the signal frequency modulation can be achieved.

Frequency DE multiplication signal of System clock also can be used as carrier signal. Divide the system clock into M signals with different frequencies. Each signal corresponds to a digital baseband signal. The baseband signal can be transmitted by the order and length of the signals with different frequencies.

\section{FSK Modulation}

Frequency shift keying (FSK), also known as frequency selection method, the following figure is the principle of its realization.

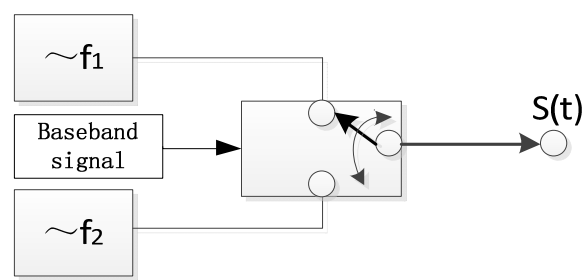

Figure 1. Principle of FSK

It has two independent oscillators and one conversion switch. The switch is controlled by the digital baseband signal to select the high frequency oscillation signal with different frequency. The FSK signal produced by keying method has high frequency stability and no transition frequency.

When the input is 0 or 1 , the modulation signal is as follows:

$$
S(t)= \begin{cases}A \cos \pi f_{1} t, & \text { send } 0 \\ A \cos \pi f_{2} t, & \text { send } 1\end{cases}
$$

$\mathrm{M}$ frequencies modulation has $\mathrm{M}$ different carrier frequencies corresponding to $\mathrm{M}$ kinds of digital information. The following figure is the principle of its realization. 


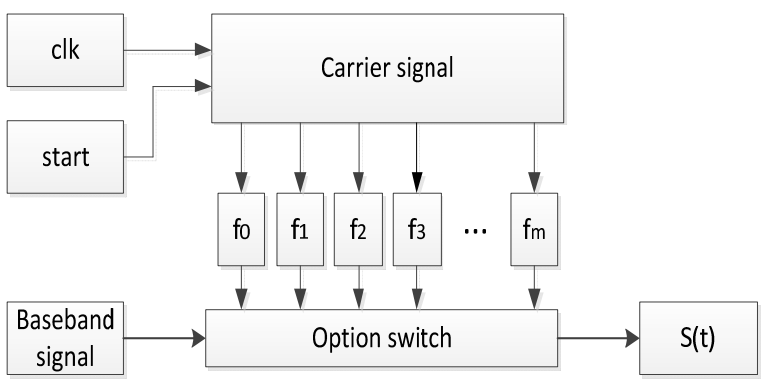

Figure 2. Principle of MFSK modulation

When the input is $0,1,2,3$, me thodulation signal is as follows:

$$
S(t)=\left\{\begin{array}{cc}
A \cos \pi f_{0} t, & \text { send } 0 \\
A \cos \pi f_{1} t, & \text { send } 1 \\
\vdots & \vdots \\
A \cos \pi f_{m} t, & \text { send } m
\end{array}\right.
$$

A plurality of sine waves of different frequencies represent different digital signals, and only one frequency is transmitted in a symbol time [3] [4]...

The following figures are multi-frequency modulation signal simulations (simulation software is Modalism).

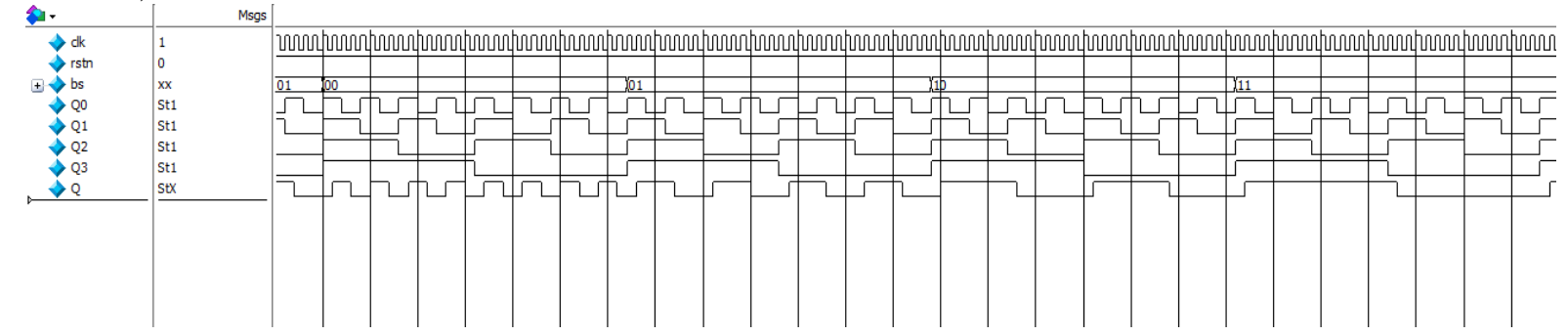

Figure 3. MFSK modulation
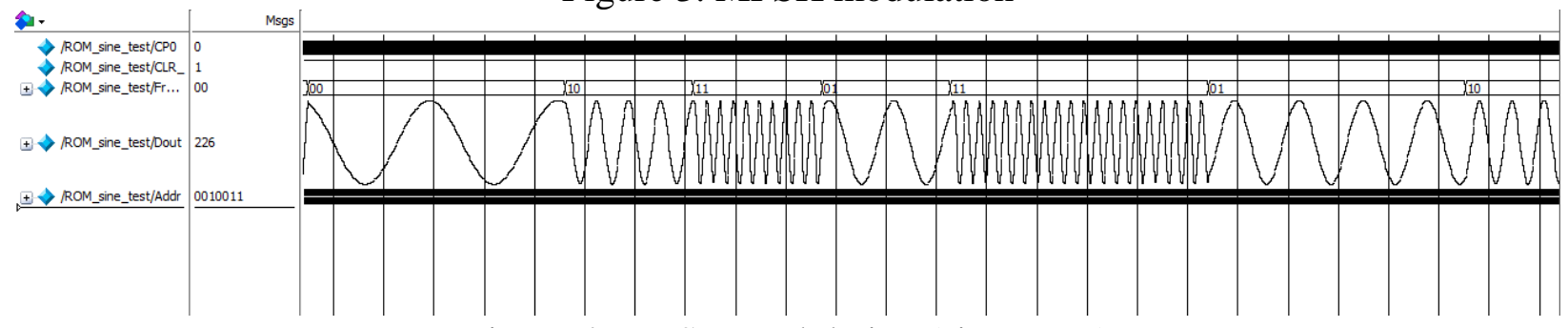

Figure 4. MFSK modulation (sine wave)

In the figure 3, System clock signal divides into 4 different frequency signals Q0, Q1, Q2, and Q3. Digital baseband signal is bs. It consists of 0-3. Q is modulated signal.

In the figure 4, each baseband signal corresponds to a frequency sine wave carrier signal. The two figures show the same principle except different carriers. The simulation result shows that the design achieves the effect of multi-frequency modulation.

\section{FSK Demodulation}

There are many kinds of commonly used demodulation methods of FSK signal. For example,

a. Synchronous (coherent) demodulation: In the synchronous demodulation, there are upper and lower two branches. The input FSK signal passes through the two band-pass filters into the upper and lower signals. The decision device needs to compare the upper and lower two branches, and finally get the baseband digital signal [6].

b. Zero crossing detection: Two narrow band filters are used to filter out the high frequency pulses of $\mathrm{f} 1$ and $\mathrm{f} 2$, and the envelope is extracted after the envelope detection. The two outputs are sent to the sampling decision device at the same time to determine the output baseband digital signal [7]. 
c. Differential detection: It is the use of signal waveform in the unit time and the number of times the number of horizontal axis to determine the frequency of the signal [9] [10].

In this paper, a simple and convenient method is used to realize MFSK signal demodulation. The most important demodulation process is execution of judgment task. The following figure is the principle of FSK demodulation.

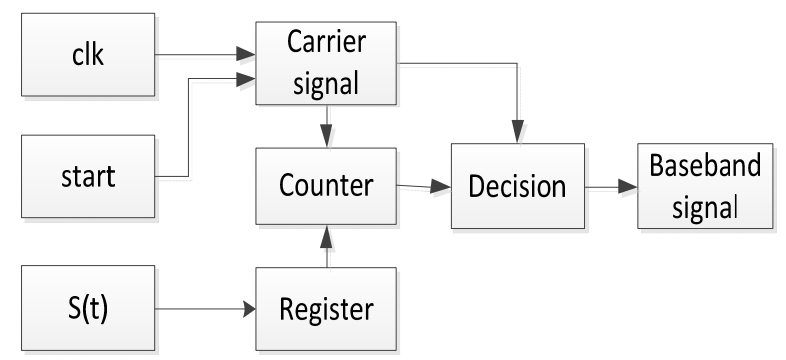

Figure 5. Principle of MFSK demodulation

Divide modulation signal into groups. Send them into the register in proper order. When the carrier is frequency DE multiplication signal of the system clock, count the number of high levels of each group. When the carrier is sine wave, then count the number of peaks of each group. According to the number of high levels or peaks, the sequence corresponding to the baseband signal can be determined [3] [5]. For example, there is 8 high levels, then the corresponding baseband sequence is 0 ; there is 1 peak, then the corresponding baseband sequence is 0 ; there are 2 peaks, corresponding to the baseband sequence is 1 .

The following figures are multi-frequency demodulation signal simulations (simulation software is Model Sim).

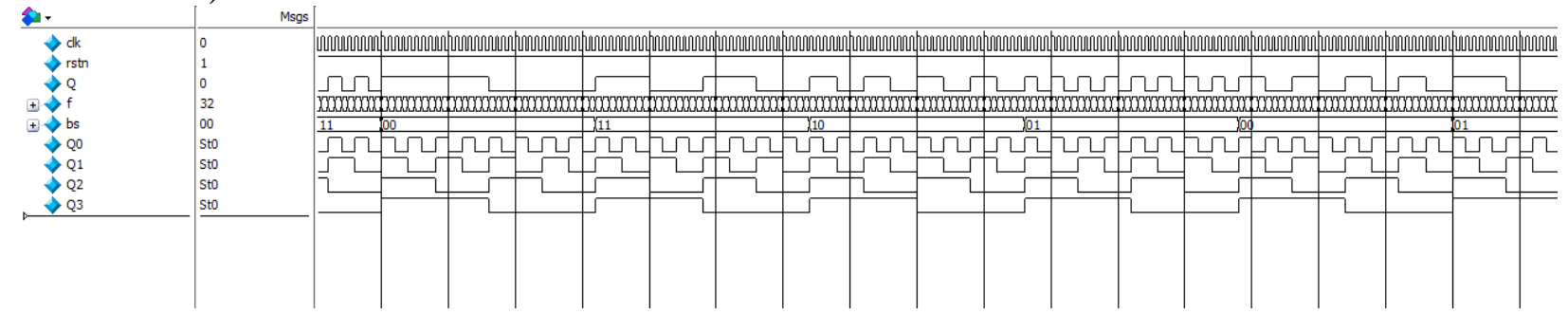

Figure 6. MFSK demodulation

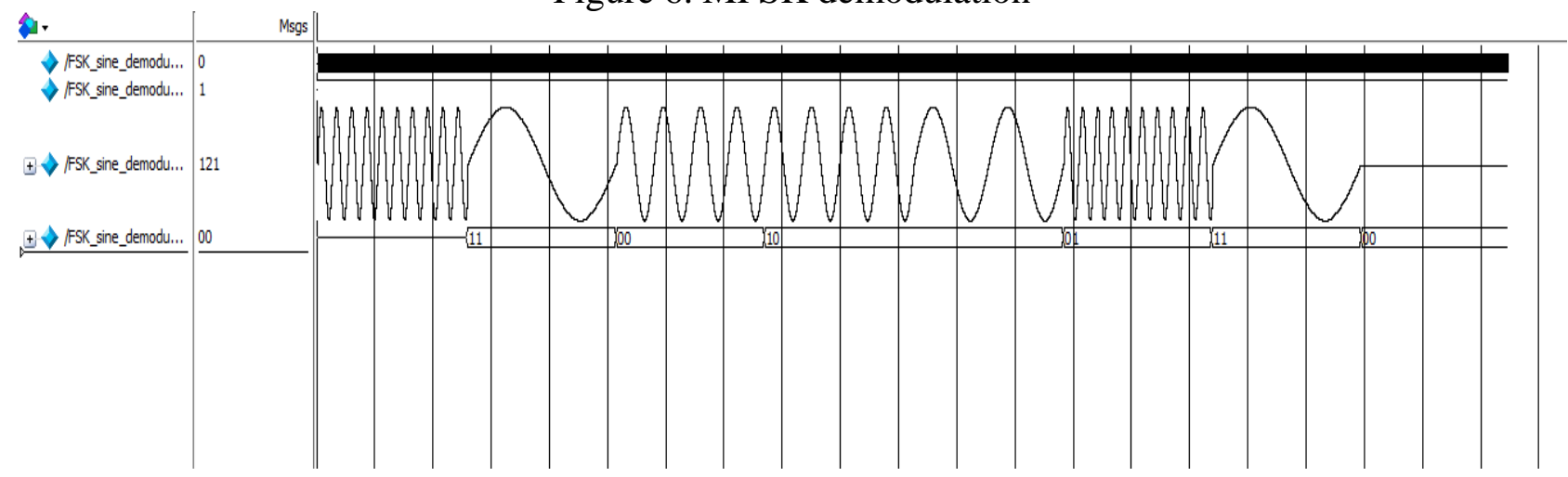

Figure 7. MFSK demodulation (sine wave)

It can be seen from the simulation results that the output of the demodulator is lagging behind the carrier input signal. Only when a group of carrier signals are input, the receiver can accurately judge the baseband signal [8].

\section{Conclusion}

In this paper, the basic principle of FSK modulation is discussed. The entire modulation and demodulation method is implemented in QUARTUS II environment using Verilog language. According to the two simulation figures, the baseband signal can be transmitted efficiently through the carrier, and accurately demodulated, which reaches design requirements. 


\section{References}

[1]. Oppenheim A V, Willsky A S, Nawaz S H. Signals \& systems (2nd Ed.) [M]. Prentice-Hall, Inc. 1996.

[2]. Bennett W R, Rice S O. Spectral Density and Autocorrelation Functions Associated with Binary Frequency-Shift Keying [J]. Bell System Technical Journal, 1963, 42 (5):2355-2385.

[3]. Palnitkar S, Verilog H. A Guide to Digital Design and Synthesis [J]. 1996, 33.

[4]. Mano M M, Ciletti M D. Digital design: with an introduction to Verilog HDL [M]. Pearson, 2013.

[5]. IEEE Standard Hardware Description Language Based on the Verilog (R) Hardware Description Language [J]. 1996:1-688.

[6]. Yi L I. Modulation Property Comparison and its Matlab/Simulink Simulation of 2FSK, MSK and GMSK [J]. 2012.

[7]. Haitao W U, Liang Y, Chen Y. Design of Full Digital FSK Modem Based on FPGA [J]. Modern Electronics Technique, 2007.

[8]. Liu F. Design and Realization of Digital IF Modulation and Demodulation System [J]. Modern Electronics Technique, 2011.

[9]. Zhang J J, Lin X U, Cheng Z X. The Demodulation of Multi-mode Communication Signals and the Adaptive Symbol Synchronization Technique Based on FPGA [J]. Telecommunication Engineering, 2005.

[10]. Ying Y P, Jian-Feng $\mathrm{X}$ U, Chen $\mathrm{W}$ J. Design and implementation of 2FSK modulation-demodulation system based on FPGA [J] . Journal of Zhejiang University of Technology, 2010. 